\title{
Cooperation in multiorganization matching
}

\author{
Laurent Gourvès $^{1,2}$, Jérôme Monnot ${ }^{1,2}$, and Fanny Pascual ${ }^{3}$ \\ 1. CNRS, UMR 7024, F-75775 Paris, France \\ 2. Université de Paris-Dauphine, LAMSADE, F-75775 Paris, France \\ 3. LIP6, Université Pierre et Marie Curie - Paris 6, 104 avenue \\ du Président Kennedy, 75016 Paris, France \\ \{laurent.gourves, monnot\}@lamsade.dauphine.fr, fanny.pascual@lip6.fr
}

\begin{abstract}
We study a problem involving a set of organizations. Each organization has its own pool of clients who either supply or demand one unit of an indivisible product. Knowing the profit induced by each buyerseller pair, an organization's task is to conduct such transactions within its database of clients in order to maximize the amount of the transactions. Inter-organizations transactions are allowed: in this situation, two clients from distinct organizations can trade and their organizations share the induced profit. Since maximizing the overall profit leads to unacceptable situations where an organization can be penalized, we study the problem of maximizing the overall profit such that no organization gets less than it can obtain on its own. Complexity results, an approximation algorithm and a matching inapproximation bound are given.
\end{abstract}

Keywords: Assignment problem; Cooperation; Complexity; Approximation

\section{Introduction}

We are given a two-sided assignment market $(B, S, A)$ defined by a set of buyers $B$, a disjoint set of sellers $S$, and a nonnegative matrix $A=\left(a_{i j}\right)_{(i, j) \in B \times S}$ where $a_{i j}$ represents a profit if the pair $(i, j) \in B \times S$ trade. In this market products come in indivisible units, and each participant either supplies or demands exactly one unit. The units do need not be alike and the same unit may have different values for different participants.

We study a problem involving a set of organizations $\left\{O_{1}, \ldots, O_{q}\right\}$ which forms a partition of the market. A buyer (resp. seller) is a client of exactly one organization. It is assumed that for every transaction $(i, j)$, organizations of $i$ and $j$ make a profit $a_{i j}$, i.e. a commission. The seller's organization receives $p_{s} a_{i j}$ while the buyer's organization gets $p_{b} a_{i j}$, where $p_{s}$ and $p_{b}$ are fixed numbers between 0 and 1 and such that $p_{b}+p_{s}=1$. We assume without loss of generality that $0 \leq p_{b} \leq p_{s} \leq 1$.

In this model, buyers and sellers do not make pairs by themselves, but these pairs are formed by their organizations. Each organization acts as a selfish agent who only knows its list of clients and only cares about its profit. Thus, each organization $O_{i}$ shall maximize the weight of a matching on $G$ induced by the vertices 
of $O_{i}$ (this task can be done in polynomial time). However the global profit can be better if transactions between clients of distinct organizations are allowed. This leads to a situation of cooperation where the agents accept to disclose their lists of clients by reporting them to a trusted entity. This trusted entity can conduct transactions between a buyer and a seller from distinct organizations, and of course, it can also do it for two clients of the same organization. The trusted entity shall maximize the collective profits. However, maximizing the collective profits by returning a maximum weight matching may lead to unacceptable situations: each organization is selfish so it does not want to cooperate if its profit is worse than it could obtain on its own. The optimization problem faced by the trusted entity is then to maximize the collective profit so that no organization is penalized.

\subsection{The multiorganization assignment problem}

The market is modelled with a weighted bipartite graph $G=(B, S ; E ; w)$ and $q$ sets (or organizations) $O_{1}, \ldots, O_{q}$ forming a partition of $B \cup S$. Every buyer (resp. seller) is represented by a vertex in $B$ (resp. $S$ ), $E \subseteq B \times S$ is the edge set representing pairs and $w: E \rightarrow \mathbb{R}_{+}$is a nonnegative weight function. The subgraph of $G$ induced by $O_{i}$ is denoted by $G_{i}$. We have $G_{i}=\left(B_{i}, S_{i} ; E_{i}, w\right)$ where $B_{i}=B \cap O_{i}$ and $S_{i}=S \cap O_{i}$. A set $M \subseteq E$ is an assignment (or a matching) iff each vertex in $(B, S ; M, w)$ has degree at most one. The weight of an assignment $M$ (i.e. the sum of the weights of its edges) is denoted by $w(M)$, and the profit of organization $O_{i}$ in $M$ is denoted by $w_{i}(M)$ and defined as

$$
w_{i}(M)=\sum_{\left\{[b, s] \in M:(b, s) \in B_{i} \times S\right\}} p_{b} w([b, s])+\sum_{\left\{[b, s] \in M:(b, s) \in B \times S_{i}\right\}} p_{s} w([b, s])
$$

where $p_{s}$ and $p_{b}$ are two nonnegative rational numbers such that $p_{s}+p_{b}=1$ and $0 \leq p_{b} \leq p_{s} \leq 1$.

We say that an edge whose endpoints are in the same organization (resp. in distinct organizations) is internal (resp. shared). The maximum weight matching of $G$ reduced to its internal edges is denoted by $\tilde{M}$. Let $\tilde{M}_{i}$ be the restriction of $\tilde{M}$ to $G_{i}$. The multiorganization assignment problem (MOA for short) is to find a maximum weight matching $M$ of $G$ such that $w_{i}(M) \geq w_{i}(\tilde{M})$ for all $i \in\{1, \ldots, q\}$. As a notation, $M^{*}$ denotes a maximum weight matching of $G$ whereas $M_{\text {cont }}^{*}$ is an optimum for MOA.

\subsection{Applications}

We give here two applications where MOA arises.

The "agencies problem". Each organization has its own pool of sellers $(S)$ and buyers $(B)$ who either supply or demand one unit of an indivisible product. Consider for example that organizations are real estate agencies. Each organization receives a commission on each transaction it deals, and its goal is to 
maximize its profit. Therefore each organization accepts the assignment given by a trusted entity if and only if its profit is at most equal to the profit it would have had without sharing its file with the other organizations. The overall aim is then to find an assignment which maximizes the total amount of transactions done, while guaranting that no organization decreases its profit by sharing its file.

A scheduling example. Each organization (which can be a university, laboratory, etc.) owns unit tasks (given by its users), and several (possibly different) machines. During some given time slots, the machines are available to schedule the tasks of the users. Each user gives her preferences for a given machine and a given time slot. These preferences are represented by integers $\left(a_{i j}\right)$ between 0 (a task cannot be scheduled on this machine at this time), and a given upper bound. The goal of each organization is to maximize the average satisfaction of its users, represented by the sum of the satisfactions of its users divided by the number of users, in the returned assignment. Therefore an organization will accept a multiorganization assignment if and only if the average satisfaction of its users is at least as high as when the organization accepts only the tasks from its users. Here, an unmatched user's satisfaction is 0 . This corresponds to MOA when $S$ is the set of users, $B$ the set of couples (time slot, machine), $p_{s}=1$ and $p_{b}=0$.

\subsection{Related work}

The multi-organization assignment problem is a variant of the old assignment problem (see [11] for a recent survey). Besides its combinatorial structure, MOA involves self-interested agents whose cooperation can lead to significant improvements but a solution is feasible only if it does not arm any local utility.

Non cooperative game theory studies situations involving several players whose selfish actions affect each others [10]. In Tucker's prisoner's dilemma, two players can either cooperate (C), i.e. stay loyal to the other prisoner,

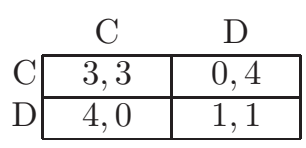

the prisoner's dilemma or defect (D), i.e. agree to testify against the other.

A social optimum is reached if both play $C$ but the situation where both prisoners defect is the only stable situation (a Nash equilibrium). In fact, the game designer of the prisoner's dilemma filled the payoff matrix in way that any prisoner has incentive to defect. MOA models the opposite situation where the game designer tries to fill the payoff matrix such that each organization's (weakly) dominant strategy is to cooperate, i.e. disclose her list of clients and follow the trusted entity. The game designer has to compute a Nash equilibrium (a stable matching) that optimizes the social welfare (total profit).

The maximum weight matching $M^{*}$ is sometimes unstable because the organizations are selfish. Then, one has to consider a different optimum $M_{\text {cont }}^{*}$ which is the maximum weight Nash equilibrium (no organization can increase its profit by using its own maximum weight matching instead of the solution 
returned by the trusted entity). Interestingly, a theoretical measure of this loss of profit due to the selfishness of the organizations exists. Known as the price of stability (PoS) [1], it is defined as the (worst case) ratio between the most socially valuable state and the worth of the best Nash equilibrium. For MOA, $\mathrm{PoS}=w\left(M_{\text {cont }}^{*}\right) / w\left(M^{*}\right)$.

MOA is also related to cooperative game theory [10]. A central issue in this field is to allocate the worth of a coalition to its members. Shapley and Shubik associate to any two-sided assignment market $(B, S, A)$ a cooperative game with transferable utility (the assignment game) and show that its core is nonempty, and has a lattice structure [12].

MOA is close in spirit to other works which study, at an algorithmic level, how to make organizations cooperate. In [9], the authors study a scheduling problem involving several organizations. Each of them has a set of jobs to be completed as early as possible and its own set of processors. A selfish schedule is such that the processors only execute jobs of their owner. The authors propose an algorithm which returns schedules with good makespans and in which the organizations cooperate without being penalyzed. In $[6,5]$, the authors study the selfish distributed replication problem. This problem involves several nodes of a network whose task is to fetch electronic contents (objects) located at distant servers. Instead of taking an object from its server at each request, the nodes can save time by making a local copy. An intermediate strategy is to get an object from another node which is closer than the server. The optimization problem is to fill the (bounded) memory of each node in order to minimize the overall expected response time. Since an optimum solution can be unacceptable to selfish nodes (e.g. a node's memory is filled with objects that it rarely requests), the authors of [5] propose equilibrium placement strategies where no one is penalyzed.

\subsection{Contribution}

We investigate the computational complexity of MOA in Section 2. In particular, we show that the problem is strongly NP-hard if the number of organizations if not fixed. It is weakly NP-hard for two organizations. A possible proof of strong NP-hardness for a fixed number of organizations is discussed and some pseudo-polynomial and polynomial cases are given as well. We provide an approximation algorithm with performance guarantee $p_{b}$ and a matching proof of inapproximation in Section 3. We also show in this section that the price of stability of MOA is $p_{b}$. Section 4 is devoted to generalizations of MOA and also generalizations of the results of this article. We conclude in Section 5 .

Our results apply for any values of $p_{s}$ and $p_{b}$ such that $0 \leq p_{b} \leq p_{s} \leq 1$ and $p_{b}+p_{s}=1$. Some proofs are put in an Appendix due to space limitation.

\section{Complexity results}

We prove that MOA is strongly NP-hard in the general case, even if the weights are polynomially bounded. We also show that the restriction of MOA to 2 orga- 
nizations is weakly NP-hard. Next we show pseudopolynomial and polynomial cases.

\subsection{Computationaly hard cases}

Given a positive profit $P$ and an instance of MOA, the decision version asks whether the instance admits a matching $M$ such that $\forall_{i \in\{1, \ldots, q\}} w_{i}(M) \geq w\left(\tilde{M}_{i}\right)$ and $w(M) \geq P$.

Theorem 1. The decision version of MOA is strongly NP-complete.

We make a reduction from 3-PARTITION which is strongly NP-complete (problem [SP15] in [3]).

Theorem 2. The decision version of MOA is NP-complete, even if there are 2 organizations and the underlying graph is of maximum degree 2.

Proof. Let $p_{s}$ and $p_{b}$ be two reals such that $1 \geq p_{s} \geq p_{b} \geq 0$ and $p_{s}+p_{b}=1$. The reduction is done from PARTITION: given a set $\left\{a_{1}, \ldots, a_{n}\right\}$ of $n$ integers such that $\sum_{i=1}^{n} a_{i}=2 W$, decide whether $J \subset\{1, \ldots, n\}$ such that $\sum_{j \in J} a_{j}=W$ exists. PARTITION is known to be NP-complete (problem [SP12] in [3]).

From $I$, instance of PARTITION, we build $I^{\prime}$, instance of MOA by the following way:

- we are given 2 organizations $O_{1}$ and $O_{2}$

- $O_{1}$ has $n+1$ sellers and $n+1$ buyers respectively denoted by $s_{1, i}$ and $b_{1, i}$ for $i=1, \ldots, n+1$

- $O_{2}$ has also $n+1$ buyers and $n+1$ sellers respectively denoted by $b_{2, i}$ and $s_{2, i}$ for $i=1, \ldots, n+1$

- The edge set of the underlying graph is given by $\left\{\left[b_{1, i}, s_{1, i}\right],\left[s_{1, i}, b_{2, i}\right],\left[b_{1, i}, s_{2, i}\right]\right.$ : $i=1, \ldots, n\} \cup\left\{\left[s_{1, n+1}, b_{2, n+1}\right],\left[b_{2, n+1}, s_{2, n+1}\right],\left[s_{2, n+1}, b_{1, n+1}\right]\right\}$

The weight are defined by:

- $w\left(\left[b_{1, i}, s_{1, i}\right]\right)=6 a_{i}$ and $w\left(\left[b_{2, i}, s_{1, i}\right]\right)=w\left(\left[s_{2, i}, b_{1, i}\right]\right)=3 a_{i}$ for $i=1, \ldots, n$

- $w\left(\left[b_{2, n+1}, s_{2, n+1}\right]\right)=6 W$ and $w\left(\left[s_{1, n+1}, b_{2, n+1}\right]\right)=w\left(\left[b_{1, n+1}, s_{2, n+1}\right]\right)=$ $3 W+1$

The underlying graph is made of a collection of $n+1$ disjoint paths of length 3. Figure 1 gives an illustration of this construction.

Organization $O_{1}$ can make a profit $w_{1}(\tilde{M})=\left(p_{s}+p_{b}\right) \sum_{i=1}^{n} 6 a_{i}=12 W$ if it works alone. The local profit of organization $O_{2}$ is $w_{2}(\tilde{M})=\left(p_{s}+p_{b}\right) 6 \mathrm{~W}=6 \mathrm{~W}$. Thus, globally, the weight of this matching is $18 \mathrm{~W}$.

We affirm that $I^{\prime}$ admits a feasible assignment $\hat{M}$ such that $w(\hat{M}) \geq 18 W+2$ if and only if $I$ admits a set $J \subseteq\{1, \ldots, n\}$ with $\sum_{j \in J} a_{j}=W$.

Let $J$ be a subset of $\{1, \ldots, n\}$ such that $\sum_{j \in J} a_{j}=W$ (and then, $\sum_{j \notin J} a_{j}=$ $W)$. We build the assignment $\hat{M}$ as follows: $\hat{M}=\left\{\left[b_{2, j}, s_{1, j}\right],\left[s_{2, j}, b_{1, j}\right]: j \in\right.$ 


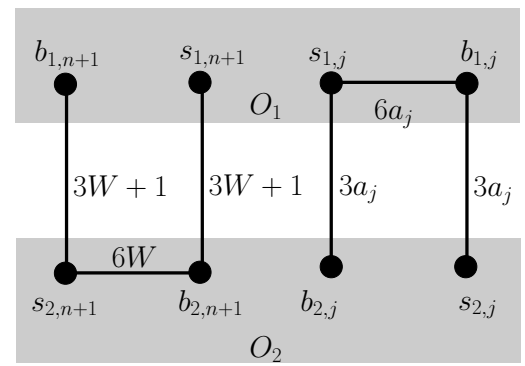

Fig. 1. The construction of $I^{\prime}$.

$J\} \cup\left\{\left[b_{1, j}, s_{1, j}\right]: j \notin J\right\} \cup\left\{\left[s_{1, n+1}, b_{2, n+1}\right],\left[b_{1, n+1}, s_{2, n+1}\right]\right\}$

Clearly, the cost of $\hat{M}$ is given by $w(\hat{M})=18 W+2$. Now, let us verify that $\hat{M}$ is a feasible solution. The local profit of organization $O_{1}$ is $\left(p_{s}+p_{b}\right) \sum_{j \notin J} 6 a_{j}+$ $\left(p_{s}+p_{b}\right) \sum_{j \in J} 3 a_{j}+\left(p_{s}+p_{b}\right)(3 W+1)=12 W+1 \geq w_{1}(\tilde{M})$ whereas the profit of organization $\mathrm{O}_{2}$ becomes $\left(p_{s}+p_{b}\right) \sum_{j \in J} 3 a_{j}+\left(p_{s}+p_{b}\right)(3 W+1)=6 W+1 \geq$ $w_{2}(\tilde{M})$.

Conversely, let $\hat{M}$ be a feasible assignment such that $w(\hat{M}) \geq 18 W+2$. Observe that the following property which can be easily proved.

Property 1. Any feasible solution of MOA can be supposed maximal with respect to the inclusion.

Now, remark that $\hat{M}$ necessarily contains the edges $\left[s_{1, n+1}, b_{2, n+1}\right]$ and $\left[b_{1, n+1}, s_{2, n+1}\right]$ since on the one hand, the weight of any maximal matching on the graph induced by all vertices except $\left\{s_{1, n+1}, s_{2, n+1}, b_{1, n+1}, b_{2, n+1}\right\}$ is $12 W$, and on the other hand $w\left(\left[b_{2, n+1}, s_{2, n+1}\right]\right)=6 W$. Thus, $\hat{M}$ must contain some edges $\left[b_{2, j}, s_{1, j}\right]$ or $\left[b_{1, j}, s_{2, j}\right]$ in order to compensate the loss of edge $\left[b_{2, n+1}, s_{2, n+1}\right]$. Let $J=\left\{j \leq n:\left[b_{2, j}, s_{1, j}\right] \in \hat{M}\right\}$. By property $1, \hat{M}$ is completely described by $\hat{M}=$ $\left\{\left[b_{2, j}, s_{1, j}\right],\left[b_{1, j}, s_{2, j}\right]: j \in J\right\} \cup\left\{\left[b_{1, j}, s_{1, j}\right]: j \notin J\right\} \cup\left\{\left[s_{1, n+1}, b_{2, n+1}\right],\left[b_{1, n+1}, s_{2, n+1}\right]\right\}$.

The profit of organization $O_{2}$ is $\left(p_{s}+p_{b}\right) \sum_{j \in J} 3 a_{j}+\left(p_{s}+p_{b}\right)(3 W+1)=$ $3 \sum_{j \in J} a_{j}+3 W+1$. Since that profit is at least $w_{2}(\tilde{M})=6 W$, we deduce that $\sum_{j \in J} a_{j} \geq W-\frac{1}{3}$. Finally, $\sum_{j \in J} a_{j}$ must be an integer, so $\sum_{j \in J} a_{j} \geq W$. On the other hand, the profit of organization $O_{1}$ is given by $\left(p_{s}+p_{b}\right) \sum_{j \notin J} 6 a_{j}+\left(p_{s}+\right.$ $\left.p_{b}\right) \sum_{j \in J} 3 a_{j}+\left(p_{s}+p_{b}\right)(3 W+1)=6 \sum_{j=1}^{n} a_{j}-3 \sum_{j \in J} a_{j}+3 W+1$. This quantity must be at least $w_{1}(\tilde{M})=6 \sum_{j=1}^{n} a_{j}$. Since $\sum_{j \in J} a_{j}$ is an integer, we obtain $\sum_{j \in J} a_{j} \leq W$. In conclusion, $\sum_{j \in J} a_{j}=W$ which means that $\left\{a_{1}, \ldots, a_{n}\right\}$ can be partitioned into two sets of weight $W$.

Is MOA strongly NP-complete for two organizations? We were not able to answer but we can relate the question to another one stated more than 25 years 
ago and still open: Is the exact weighted perfect matching problem in bipartite graphs strongly NP-complete?

Given a graph whose edges have an integer weight and given a bound $W$, ExAстPM is to decide whether the graph contains a perfect matching $M$ of total weight exactly $W[2,4,7,8]$. Papadimitriou and Yannakakis [8] prove that ExaCTPM is (weakly) NP-complete in bipartite graphs. Barahona and Pulleyblank [2] propose a pseudopolynomial algorithm in the case of planar graphs and Karzanov [4] gives a polynomial algorithm when the graph is either complete or complete bipartite and the weights are restricted to 0 or 1. Mulmuley, Vazirani and Vazirani [7] show that EXACTPM has a randomized pseudo-polynomialtime algorithm. However, the deterministic complexity of this problem remains unsettled, even for bipartite graphs. (Papadimitriou and Yannakakis conjectured that it is strongly NP-complete [8]).

EXACTPM is an auto-reducible problem, that is find a perfect matching of weight $W$ is polynomially equivalent to decide whether such a matching exists.

Here, we prove that there is a Turing reduction from MOA when there are 2 organizations to ExACTPM. Thus, we conclude that if MOA with 2 organizations is strongly NP-complete then ExACTPM is also strongly NP-complete in bipartite graphs. Notice that this result also holds when there is a constant number of organizations.

Proposition 1. If EXАСТPM is polynomial in bipartite graphs when weights are polynomially bounded, then MOA with 2 organizations and weights polynomially bounded is polynomial for every values of $p_{s}, p_{b}$ such that $1 \geq p_{s} \geq p_{b} \geq 0$ and $p_{s}+p_{b}=1$.

We can similarly show:

Proposition 2. MOA with a constant number of organizations can be solved within pseudopolynomial time when the underlying graph has a maximum degree 2.

\subsection{Polynomial cases}

MOA is trivially polynomial when there is a unique organization or when the underlying graph is of maximum degree 1 . Furthermore an exhaustive search can efficiently solve the problem if the underlying graph $G=(V, E)$ contains $\mathcal{O}(\log |E|)$ shared edges. Let $\mathrm{MOA}_{0,1}$ be the subcase where $w([i, j]) \in\{0,1\}$ for all $(i, j) \in B \times S$. We prove that an optimum to $\mathrm{MOA}_{0,1}$ is a maximum cardinality assignment of the underlying graph though a maximum cardinality assignment is not necessarily a solution of $\mathrm{MOA}_{0,1}$.

Theorem 3. $\mathrm{MOA}_{0,1}$ is polynomial.

Sketch of proof. The proof is based on the following algorithm. Start with $\tilde{M}$, increase its size with augmenting alternating paths while it is possible and denote by $\hat{M}$ the resulting matching. We mainly prove that improving a matching via 
an augmenting alternating path is monotone, i.e. the profit of any organization cannot decrease. At the beginning of the algorithm, the current matching $\tilde{M}$ is a feasible solution of the problem. The resulting matching $\hat{M}$ is also feasible by the monotonicity. Finally $\hat{M}$ is optimal (maximum cardinality) since no more augmenting alternating path exists.

\section{Approximation}

Recall that $p_{s}$ and $p_{b}$ are any values such that $0 \leq p_{b} \leq p_{s} \leq 1$ and $p_{s}+p_{b}=1$. We start by the following property (proof in Appendix).

Property 2. $w_{i}\left(M^{*}\right) \geq p_{b} w\left(\tilde{M}_{i}\right)$, and this bound is asymptotically tight.

Let us consider algorithm ApPROX given below.

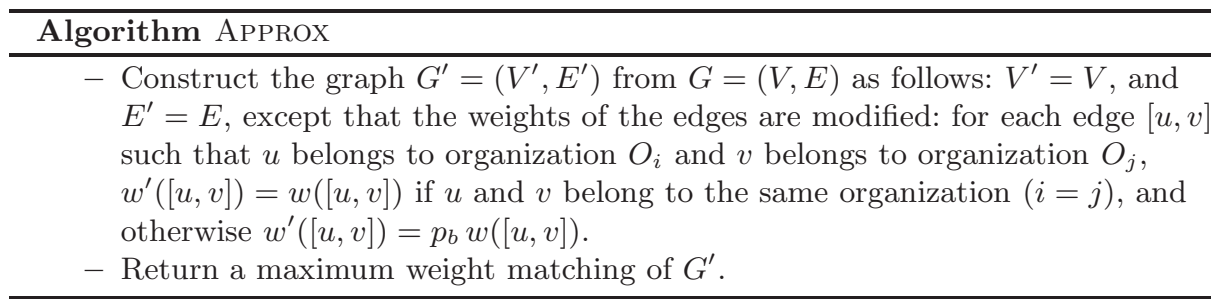

Theorem 4. APPROX is a pb-approximate algorithm for MOA, and this bound is asympotically tight.

Proof. Let $p_{s}, p_{b}$ be 2 numbers such that $1 \geq p_{s} \geq p_{b} \geq 0$ and $p_{s}+p_{b}=1$. Let $M$ be a matching returned by algorithm ApPRox on graph $G$. We first show that the profit of each organization $O_{i}$ in $M$ is at least $w\left(\tilde{M}_{i}\right)$, and thus that $M$ is a solution of MOA.

Let $M^{i n t(i)}$ be the set of edges of $M$ whose both endpoints belong to $O_{i}$, and let $M^{\operatorname{ext}(i)}$ be the set of edges of $M$ whose exactly one endpoint belongs to $O_{i}$. Since $M$ is a maximum weight matching of $G^{\prime}, w^{\prime}\left(M^{i n t(i)}\right)+w^{\prime}\left(M^{\operatorname{ext}(i)}\right) \geq$ $w\left(\tilde{M}_{i}\right)$, otherwise we could have a matching with a larger weight by replacing the edges of $\left(M^{i n t(i)} \cup M^{\operatorname{ext}(i)}\right)$ in $M$ by the edges of $\tilde{M}_{i}$. Thus the profit of $O_{i}$ is at least $\left.w\left(M^{\text {int }(i)}\right)+p_{b} w\left(M^{\operatorname{ext}(i)}\right)=w^{\prime}\left(M^{\text {int }(i)}\right)+w^{\prime}\left(M^{\operatorname{ext}(i)}\right)\right) \geq w\left(\tilde{M}_{i}\right)$.

Let us now show that Approx is $p_{b}$-approximate. The edges of $G^{\prime}$ are the same that the ones of $G$, except that the weight of some of them has been multiplied by $p_{b}<1$. Thus $M$, which is a maximum weight matching of $G^{\prime}$, has a weight $w(M) \geq p_{b} w\left(M^{*}\right) \geq p_{b} w\left(M_{\text {cont }}^{*}\right)$.

Let us show that this bound is asymptotically tight, by considering the following instance. Here, we assumed $p_{b}>0$. Recall that $p_{b} \leq 1 / 2$ since $1 \geq$ $p_{s} \geq p_{b} \geq 0$. Let $\varepsilon>0$ such that $\varepsilon<1 / p_{b}-1$. There are two organizations, organization $O_{1}$, which owns two vertices $b_{1}$ and $s_{1}$, linked by an edge 
of weight 1 , and organization $O_{2}$, which owns two vertices $b_{2}$ and $s_{2}$, linked by an edge of weight 1 . There are two shared edges, between $b_{1}$ and $s_{2}$, and between $b_{2}$ and $s_{1}$ : both edges have weight $\frac{1}{p_{b}}-\varepsilon$. Algorithm Approx returns the matching $M=\left\{\left[b_{1}, s_{1}\right],\left[b_{2}, s_{2}\right]\right\}$ with weight 2 in $G^{\prime}$ because the weight of $\left\{\left[b_{1}, s_{2}\right],\left[b_{2}, s_{1}\right]\right\}$ in $G^{\prime}$ is $2\left(1-p_{b} \varepsilon\right)<2$. The optimal solution would have been $M_{\text {cont }}^{*}=\left\{\left[b_{1}, s_{2}\right],\left[b_{2}, s_{1}\right]\right\}$. The ratio between the weights of these two solutions is $\frac{w(M)}{w\left(M_{\text {cont }}^{*}\right)}=\frac{2}{2 / p_{b}-2 \varepsilon}$, which tends towards $p_{b}$ when $\varepsilon$ tends towards 0 .

Theorem 4 implies that the price of stability of MOA defined as $w\left(M_{c o n t}^{*}\right) / w\left(M^{*}\right)$ is at least $p_{b}$. In fact, we are able to prove that $\mathrm{PoS}=p_{b}$.

Proposition 3. The price of stability is $p_{b}$.

We can prove that Theorem 4 is best possible if $\mathbf{P} \neq \mathbf{N P}$, i.e. we cannot obtain a $\left(p_{b}+\varepsilon\right)$-approximation for all $\varepsilon>0$. Actually, we prove a slightly stronger result.

Theorem 5. For any polynomial $P$, it is $\mathbf{N P}$-hard to obtain $a\left(p_{b}+\frac{1}{\Theta\left(2^{P(n)}\right)}\right)$ approximation for MOA where $n$ is the number of vertices, even if there are exactly 3 organizations.

Proof. We describe a gap reduction. We start with an instance of PARTITION given by a set of $n$ integers $\left\{a_{1}, \ldots, a_{n}\right\}$ such that $\sum_{i=1}^{n} a_{i}=2 W$. For any real $t>1$, we construct an instance $I_{t}$ of MOA as follows:

- we are given 3 organizations $O_{1}, O_{2}$ and $O_{3}$.

- $O_{1}$ has $n+1$ buyers and $n+1$ sellers respectively denoted by $b_{1, i}$ and $s_{1, i}$ for $i=1, \ldots, n+1$.

- $O_{2}$ has 2 buyers denoted by $b_{2,1}, b_{2, n+1}$ and $n+1$ sellers denoted by $s_{2, i}$ for $i=1, \ldots, n+1$.

- $O_{3}$ has one seller $s_{3,1}$.

- The edge set of the underlying graph is $\left\{\left[s_{1, i}, b_{1, i}\right],\left[b_{1, i}, s_{2, i}\right]: i=1, \ldots, n\right\} \cup$ $\left\{\left[s_{1, n+1}, b_{2,1}\right]\right\} \cup\left\{\left[b_{1, n+1}, s_{2, n+1}\right],\left[s_{2, n+1}, b_{2, n+1}\right],\left[b_{2, n+1}, s_{3,1}\right]\right\}$

The weights are given by:

- $w\left(\left[s_{1, i}, b_{1, i}\right]\right)=w\left(\left[b_{1, i}, s_{2, i}\right]\right)=a_{i}$ for $i=1, \ldots, n$.

- $w\left(\left[s_{1, n+1}, b_{2,1}\right]\right)=p_{s} W, w\left(\left[b_{1, n+1}, s_{2, n+1}\right]\right)=p_{s} W, w\left(\left[s_{2, n+1}, b_{2, n+1}\right]\right)=$ $t p_{b} W+2 p_{s} W$, and $w\left(\left[b_{2, n+1}, s_{3,1}\right]\right)=t W$.

An illustration of this construction is given in Figure 2.

If $t=\mathcal{O}\left(2^{P(|V|)}\right)$ where $|V|=3 n+6$ is the order of the underlying graph, then it is not difficult to see that the above construction is given within polynomial time.

The profits the organizations can make on their own are respectively $w_{1}(\tilde{M})=$ $\left(p_{s}+p_{b}\right) \sum_{i=1}^{n} a_{i}=2 W, w_{2}(\tilde{M})=\left(p_{s}+p_{b}\right)\left(t p_{b} W+2 p_{s} W\right)=t p_{b} W+2 p_{s} W$ and $w_{3}(\tilde{M})=0$. 


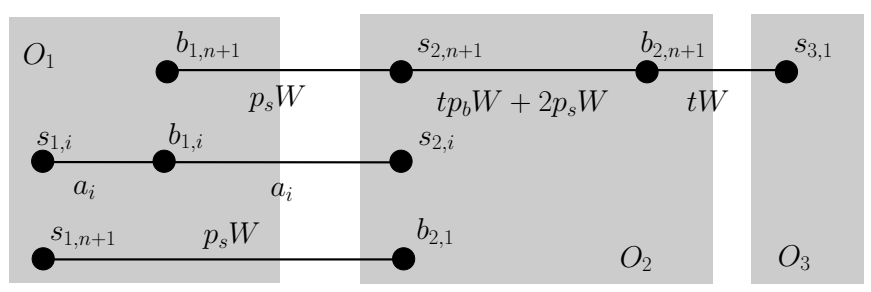

Fig. 2. The instance $I_{t}$ resulting from the above reduction

We prove that there are only two distinct values for the optimal value of MOA, that are $O P T\left(I_{t}\right)=t p_{b} W+3 p_{s} W+2 W$ or $O P T\left(I_{t}\right)=t W+2 p_{s} W+2 W$ and $O P T\left(I_{t}\right)=t W+2 p_{s} W+2 W$ if and only if $\left\{a_{1}, \ldots, a_{n}\right\}$ admits a partition.

Observe that $t W+2 p_{s} W+2 W>t p_{b} W+3 p_{s} W+2 W$ if and only if $t>1$ since $p_{b}=1-p_{s}$ and $p_{s}>0$. Let $M_{\text {cont }}^{*}$ be an optimal solution of MOA (with value $\left.O P T\left(I_{t}\right)\right)$. Let us consider two cases:

Case $\left[s_{2, n+1}, b_{2, n+1}\right] \in M_{\text {cont }}^{*}$. An optimal solution can be described by

$$
\left\{\left[s_{1, i}, b_{1, i}\right]: i=1, \ldots, n\right\} \cup\left\{\left[s_{1, n+1}, b_{2,1}\right],\left[s_{2, n+1}, b_{2, n+1}\right]\right\} .
$$

Actually, $\left[s_{1, n+1}, b_{2,1}\right] \in M_{\text {cont }}^{*}$ because $M_{\text {cont }}^{*}$ is maximal by Property 1 (cf page 6). Moreover, the weight of any maximal matching on the graph induced by $\left\{s_{1, i}, b_{1, i}, s_{2, i}: i=1, \ldots, n\right\}$ has the same value $2 W$. In this case, we get $O P T\left(I_{t}\right)=t p_{b} W+3 p_{s} W+2 W$.

Case $\left[s_{2, n+1}, b_{2, n+1}\right] \notin M_{\text {cont }}^{*}$. Edges $\left\{\left[b_{1, n+1}, s_{2, n+1}\right],\left[b_{2, n+1}, s_{3,1}\right],\left[s_{1, n+1}, b_{2,1}\right]\right\}$ belong to $M_{\text {cont }}^{*}$ by Property 1 . The contribution of these 3 edges to the profit of $O_{2}$ is $p_{s} w\left(\left[b_{1, n+1}, s_{2, n+1}\right]\right)+p_{b} w\left(\left[b_{2, n+1}, s_{3,1}\right]\right)+p_{b} w\left(\left[s_{1, n+1}, b_{2,1}\right]\right)=t p_{b} W+$ $p_{s} W<t p_{b} W+2 p_{s} W=w\left(\left[s_{2, n+1}, b_{2, n+1}\right]\right)$ since $p_{s}>0$. Hence, a subset of shared edges between $O_{1}$ and $O_{2}$ must belong to $M_{\text {cont }}^{*}$. Let $J^{*}=\{j \leq$ $\left.n:\left[b_{1, j}, s_{2, j}\right] \in M_{\text {cont }}^{*}\right\}$ be this subset. Then, $M_{\text {cont }}^{*}$ is entirely described by $\left\{\left[b_{1, n+1}, s_{2, n+1}\right],\left[b_{2, n+1}, s_{3,1}\right],\left[s_{1, n+1}, b_{2,1}\right]\right\} \cup\left\{\left[b_{1, j}, s_{2, j}\right]: j \in J^{*}\right\} \cup\left\{\left[s_{1, j}, b_{1, j},\right]:\right.$ $\left.j \notin J^{*}\right\}$.

To be feasible, $M_{\text {cont }}^{*}$ must satisfy $w_{1}\left(M_{\text {cont }}^{*}\right) \geq w\left(\tilde{M}_{1}\right)$, i.e. $\sum_{j \notin J^{*}} a_{j}+$ $p_{b} \sum_{j \in J^{*}} a_{j}+\left(p_{s}+p_{b}\right) p_{s} W \geq \sum_{j=1}^{n} a_{j}$ from which we deduce $W \geq \sum_{j \in J^{*}} a_{j}$ because $p_{b}=1-p_{s}$ and $p_{s}>0 . M^{*}$ must also satisfy $w_{2}\left(M_{\text {cont }}^{*}\right) \geq w\left(\tilde{M}_{2}\right)$, i.e. $p_{s} \sum_{j \in J^{*}} a_{j}+\left(p_{s}+p_{b}\right) p_{s} W+t p_{b} W \geq t p_{b} W+2 p_{s} W$, which is equivalent to $\sum_{j \in J^{*}} a_{j} \geq W$. Then, we obtain $\sum_{j \in J^{*}} a_{j}=\sum_{j \notin J^{*}} a_{j}=W$. On the one hand $O P T\left(I_{t}\right)=t W+2 p_{s} W+2 W$ and on the other hand $\left\{a_{1}, \ldots, a_{n}\right\}$ has a partition given by $J^{*}$.

Conversely, if $\left\{a_{1}, \ldots, a_{n}\right\}$ admits a partition then it is not difficult to prove that $O P T\left(I_{t}\right)=t W+2 p_{s} W+2 W$.

Now, assume that there is a $\left(p_{b}+\frac{1}{c 2^{P(V T)}}\right)$-approximation of MOA given within polynomial time for some $c>0$. Consider $t_{0}=5 c 2^{P(|V|)}$ and let $a p x\left(I_{t_{0}}\right)$ denote the value of the approximate solution on instance $I_{t_{0}}$. 
- $\left\{a_{1}, \ldots, a_{n}\right\}$ does not admit a partition. One has $O P T\left(I_{t_{0}}\right)=5 c 2^{P(|V|)} p_{b} W+$ $3 p_{s} W+2 W$ and then $a p x\left(I_{t_{0}}\right) \leq 5 c 2^{P(|V|)} p_{b} W+3 p_{s} W+2 W$.

- $\left\{a_{1}, \ldots, a_{n}\right\}$ admits a partition. We have $O P T\left(I_{t_{0}}\right)=5 c 2^{P(|V|)} W+2 p_{s} W+$ $2 W$. Since $\operatorname{apx}\left(I_{t_{0}}\right) \geq\left(p_{b}+\frac{1}{c^{P(|V|)}}\right) O P T\left(I_{t_{0}}\right)$ by hypothesis and $p_{s} \leq 1$, we deduce $\operatorname{apx}\left(I_{t_{0}}\right)>5 W+5 c 2^{P(|V|)} p_{b} W \geq 5 c 2^{P(|V|)} p_{b} W+3 p_{s} W+2 W$.

In conclusion, apx allows us to distinguish within polynomial time whether $\left\{a_{1}, \ldots, a_{n}\right\}$ has a partition or not, which is impossible if $\mathbf{P} \neq \mathbf{N P}$.

\section{Generalizations}

Relaxation of the selfishness of the organizations. Suppose that each organization $O_{i}$ accepts a proposed global matching if its own profit is at least $w\left(\tilde{M}_{i}\right) / x$ where $x \geq 1$ is fixed. This means that each organization accepts to divide by $x$ the profit it would have without sharing its file with the other organizations. The problem, denoted by $\operatorname{MOA}(x)$ is then to find a maximum weight matching $M$ such that $w_{i}(M) \geq w\left(\tilde{M}_{i}\right) / x$ for all $i \in\{1, \ldots, q\}$. Let $M_{\text {cont }(x)}^{*}$ denote such a maximum weight matching.

If $x=1$, an organization does not accept to reduce its profit, and this problem is the one stated in the Introduction. If $x \geq 1 / p_{b}$, the organizations accept to divide their profits by $1 / p_{b}$. Property 2 page 8 shows that in a maximum weight matching $M^{*}$, the profit of organization $O_{i}$ is at least $p_{b} w\left(\tilde{M}_{i}\right)$. Thus $M_{\text {cont }(x)}^{*}=M^{*}$. Our aim is now to solve $\operatorname{MOA}(x)$ for $1 \leq x<1 / p_{b}$. With a slight modification of the proof of Theorem 1, we can show that this problem is strongly NP-hard for each value $x$ smaller than $1 / p_{b}$, when there are at least three organizations. One can also extend Approx to a slightly modified algorithm ${ }^{1}$ $\operatorname{Approx}(x)$ and prove that it is $\left(x p_{b}\right)$-approximate algorithm for $\operatorname{MOA}(x)$ and this bound is tight. In addition, the price of stability is $x p_{b}$ for this generalization.

General graphs. One can extend MOA to general graphs when $p s=p_{b}=1 / 2$. In this case, the distinction between buyers and sellers is lost. For example, the problem has the following application: Numerous web sites offer to conduct home exchanges during holidays. The concept is simple, instead of booking expensive hotel rooms, pairs of families agree to swap their houses for a vacation. We model the situation with a graph $G=(V, E)$ whose vertices are candidates for house exchange. The vertex set is partitioned into $q$ sets/organizations $O_{1} \ldots O_{q}$. Vertices within an organization are its clients. Every edge $[a, b] \in E$ has a weight $w([a, b])$ representing the satisfaction of candidates $a$ and $b$ if they swap. Pairs are formed by the organizations which only care about the satisfaction of their clients. In case of a mixed-organizations exchange $[a, b]$, it is assumed that the satisfaction of both participants is $w([a, b]) / 2$. The problem is to maximize the collective satisfaction while no organization is penalized.

Theorems 3 to 5 and Proposition 3 (where $p_{b}$ is replaced by $1 / 2$ ) hold for general graphs since the proofs do not use the fact that $G$ is bipartite.

\footnotetext{
${ }^{1}$ The weight of shared edges is multiplied by $x p_{b}$ instead of $p_{b}$.
} 


\section{Conclusion}

We studied cooperation, at an algorithmic level, between organizations. We showed that the price of stability is $p_{b}$, and we studied the complexity of MOA. We presented polynomial cases, and showed that the problem is NP-hard in the general case. We also gave an approximation algorithm, matching the inapproximation bound when there are at least 3 organizations. There remains some open problems: is it possible to have an algorithm with a better approximation ratio when there are two organizations? Is this problem strongly NP-hard in this case (we notice that this problem is related to the open Exact Perfect Matching problem)? When we consider that each organization accepts a solution if it does not reduce its profit by a factor larger than $x$, is it possible to get an algorithm with an approximation ratio better than $x p_{b}$ ? An interesting direction would also be to study fairness issues in this problem. For example, among all the solutions of the same quality, return the one which maximizes the minimum $w_{i}\left(M_{\text {cont }}\right)-\tilde{M}_{i}$, that is the minimum increase of profit of the organizations.

\section{References}

1. E. Anshelevich, A. Dasgupta, J. M. Kleinberg, É. Tardos, T. Wexler and T. Roughgarden. The Price of Stability for Network Design with Fair Cost Allocation. In Proc. of FOCS 2004, pp. 295-304, 2004.

2. F. Barahona and W.R. Pulleyblank. Exact arborescences, matchings, and cycles, in Discrete Appl. Math. vol. 16, pp. 91-99, 1987.

3. M. R. Garey and D. S. Johnson. Computers and Intractability: A Guide to the Theory of NP-Completeness, W. H. Freeman and Company, New York, 1979.

4. A.V. Karzanov. Maximum matching of given weight in complete and complete bipartite graphs, In Cybernetics, vol. 23, pp. 8-13, 1987 (translation from Kibernetika, vol. 1, pp. 711, 1987).

5. N. Laoutaris, O. Telelis, V. Zissimopoulos and I. Stavrakakis. Distributed Selfish Replication, IEEE Trans. Parallel Distrib. Syst., vol 17(12), pp. 1401-1413, 2006.

6. A. Leff, L. Wolf, and P. S. Yu. Efficient LRU-based buffering in a LAN remote caching architecture, IEEE Trans. Parallel Distrib. Syst., vol. 7(2), pp. 191-206, 1996.

7. K. Mulmuley, U. Vazirani and V.V. Vazirani. Matching is as easy as matrix inversion, In Combinatorica, vol. 7, pp. 105-113, 1987.

8. C.H. Papadimitriou and M. Yannakakis. The complexity of restricted spanning tree problems, In J. ACM, vol. 29, pp. 285-309, 1982.

9. F. Pascual, K. Rzadca and D. Trystram. Cooperation in Multi-organization Scheduling, In Proc. of Euro-Par 07, LNCS 4641, pp. 224-233, 2007.

10. M. Osborne and A. Rubinstein. A Course in Game Theory, MIT Press, 1994.

11. D. W. Pentico. Assignment problems: A golden anniversary survey. EJOR, vol. 176, pp. 774-793, 2007.

12. L.S. Shapley and M. Shubik. The assignment game I: The core, International Journal of Game Theory, vol. 1, pp. 111-130, 1972. 


\section{Appendix}

Theorem 1 For any values of $p_{b}, p_{s}$ such that $1 \geq p_{s} \geq p_{b} \geq 0$ and $p_{s}+p_{b}=1$, the decision version of $\mathrm{MOA}$ is strongly $\mathbf{N P}$-complete.

Proof. Let $p_{s}$ and $p_{b}$ be two numbers such that $1 \geq p_{s} \geq p_{b} \geq 0$ and $p_{s}+p_{b}=1$. Given a positive profit $P$ and an instance of MOA, the decision version asks whether the instance admits a matching $M$ such that $\forall_{i \in\{1, \ldots, q\}} w_{i}(M) \geq w\left(\tilde{M}_{i}\right)$ and $w(M) \geq P$.

Given a bound $W$, a set $A=\left\{a_{1}, \ldots, a_{3 m}\right\}$ of $3 m$ positive integers such that $\sum_{i=1}^{3 m} a_{i}=m W$ and $\forall i=1, \ldots, 3 m, \frac{W}{4}<a_{i}<\frac{W}{2}$, the 3-PARTITION problem is to decide whether $A$ can be partitioned into $m$ subsets $A_{1}, A_{2}, \ldots, A_{m}$ such that the sum of the numbers in each subset is equal (thus $\sum_{a_{j} \in A_{i}} a_{j}=W$ and $\left|A_{i}\right|=3$ for all $\left.i \in\{1, \ldots, m\}\right)$. The 3 -PARTITION problem is strongly NPcomplete (problem [SP15] in [3]).

Given an instance $I$ of the 3-PARTITION problem, we build a corresponding instance $I^{\prime}$ of MOA as follows (see Figure 3 for an illustration):

- we are given $m+1$ organizations $O_{1}, \ldots, O_{m+1}$, i.e. $q=m+1$

- $O_{m+1}$ has $3 m$ buyers and $m$ sellers respectively denoted by $b_{m+1,1}$ to $b_{m+1,3 m}$ and $s_{m+1,1}$ to $s_{m+1, m}$

- for $i=1, \ldots, m$ :

- $O_{i}$ has 3 sellers denoted by $s_{i, 1}, s_{i, 2}, s_{i, 3}$ and one buyer $b_{i}$

- $w\left(\left[b_{i}, s_{i, 1}\right]\right)=w\left(\left[b_{i}, s_{m+1, i}\right]\right)=W$

- for $i, j \in\{1, \ldots, m\} \times\{1, \ldots, 3 m\}$ :

- $w\left(\left[b_{m+1, j}, s_{i, 1}\right]\right)=w\left(\left[b_{m+1, j}, s_{i, 2}\right]\right)=w\left(\left[b_{m+1, j}, s_{i, 3}\right]\right)=a_{j}$

We have $w_{i}(\tilde{M})=\left(p_{s}+p_{b}\right) W=W$ for $i=1, \ldots, m$ and $w_{m+1}(\tilde{M})=0$. We claim that $I^{\prime}$ admits a feasible assignment $M$ such that $w(M) \geq 2 m W$ if and only if $A$ admits a partition into $m$ subsets $A_{1}, A_{2}, \ldots, A_{m}$ such that $\sum_{a_{j} \in A_{i}} a_{j}=W$ and $\left|A_{i}\right|=3$ for all $i \in\{1, \ldots, m\}$.

Let $\hat{A}=\left\langle A_{1}, A_{2}, \ldots, A_{m}\right\rangle$ be a YES solution to instance $I$ of 3-PARTITION. We build a corresponding matching $\hat{M}$, solution to instance $I^{\prime}$ of MOA as follows: $\hat{M}=\emptyset$ at the beginning and for each triple $a_{x}, a_{y}, a_{z}$ of $A_{i}$, we add edges $\left[b_{m+1, x}, s_{i, 1}\right],\left[b_{m+1, y}, s_{i, 2}\right]$ and $\left[b_{m+1, z}, s_{i, 3}\right]$ to $\hat{M}$. We also add edge $\left[b_{i}, s_{m+1, i}\right]$ to $\hat{M}$ for all $i \in\{1, \ldots, m\}$.

We remark that $\hat{M}$ is a feasible assignment. Indeed, organization $O_{i}(i=$ $1, \ldots, m)$ has 4 shared edges in $\hat{M}$, that is $\left[b_{i}, s_{m+1, i}\right]$ with weight $W,\left[b_{m+1, x}, s_{i, 1}\right]$ with weight $a_{x},\left[b_{m+1, y}, s_{i, 2}\right]$ with weight $a_{y}$ and $\left[b_{m+1, z}, s_{i, 3}\right]$ with weight $a_{z}$.

Since $\hat{A}$ is a YES solution to $I$, we know that $a_{x}+a_{y}+a_{z}=W$. Hence, $w_{i}(\hat{M})=\left(p_{s}+p_{b}\right) W=w_{i}(\tilde{M})$ for $i=1, \ldots, m$. We also have $w_{m+1}(\hat{M})=$ $\left(p_{s}+p_{b}\right) m W$ since $O_{m+1}$ has $m$ shared edges of weight $W$ adjacent to its sellers and $3 m$ other shared edges of total weight $\sum_{j=1}^{3 m} a_{j}=m W$ adjacent to its buyers. This is greater than $w_{m+1}(\tilde{M})$. Thus, $\hat{M}$ is a YES solution to instance $I^{\prime}$ of the 


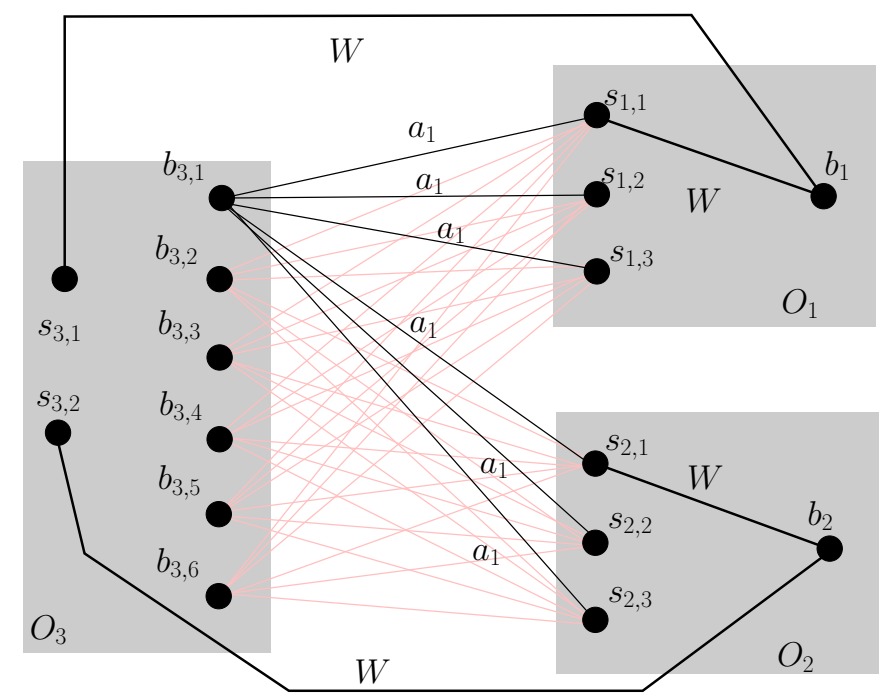

Fig. 3. Bipartite graph obtained by the transformation of an instance $A=\left\{a_{1}, \ldots, a_{6}\right\}$ of the 3-PARTITION problem where $W=\frac{1}{2} \sum_{j=1}^{6} a_{j}$. There is an edge with weight $a_{j}$ between $b_{3, j}$ and $s_{x, y}$ for all couple $x, y \in\{1,2\} \times\{1,2,3\}$. These edges are shaded in the picture except those with weight $a_{1}$.

decision version of MOA because the total profit made by the organizations is $2 m W$.

Conversely, let $\hat{M}$ be a YES solution to instance $I^{\prime}$ of the decision version of MOA with $P=2 m W$. By definition we have $w(\hat{M}) \geq 2 m W, w_{i}(\hat{M}) \geq W$ for $i=1, \ldots, m$ and $w_{m+1}(\hat{M}) \geq 0$. Observe that $\hat{M} \cap\left\{\left[b_{i}, s_{i, 1}\right] \mid i=1, \ldots, m\right\}=\emptyset$. Indeed, if $k$ edges in $\left\{\left[b_{i}, s_{i, 1}\right] \mid i=1, \ldots, m\right\}$ belong to $\hat{M}$ then the total profit would be strictly less than $2 m W$ since $w(\hat{M}) \leq k W+(m-k) W+\sum_{i=1}^{3 m} a_{i}-$ $k \min \left\{a_{i}: i=1, \ldots, 3 m\right\}<\left(2 m-\frac{k}{4}\right) W$. Furthermore, $\hat{M}$ must be perfect since otherwise $w(\hat{M})<2 m W$.

We build a partition $\hat{A}=\left\langle A_{1}, A_{2}, \ldots, A_{m}\right\rangle$, solution to instance $I$ of 3PARTITION corresponding to $\hat{M}$ as follows: for $i=1$ to $m$, put in $A_{i}$ the weight of the (shared) edges incident to $s_{i, 1}, s_{i, 2}$ and $s_{i, 3}$. One can observe that $\hat{A}$ is a feasible 3-partition of $A$. Take an organization $O_{i}(i=1, \ldots, m), 4$ shared edges are incident to its nodes in $\hat{M}$. The one incident to $b_{i}$ has weight $W$. The total weight of the three others must be at least $W$ since $w_{i}(\tilde{M})=\left(p_{s}+p_{b}\right) W$. Hence, each $A_{i}$ is assigned 3 values whose sum is at least $W$ but if this sum exceeds $W$ for at least one organization, we would have $\sum_{j=1}^{3 m} a_{j}>W m$ which is a contradiction. As a consequence, each $A_{i}$ is assigned 3 values whose sum is exactly $W$.

Theorem $3 \mathrm{MOA}_{0,1}$ is polynomial. 
Proof. Let $M$ be an assignment on an unweighted bipartite graph $G=(B, S ; E)$. Recall that a path in $G$ is alternating with respect to $M$ if it alternates edges of $M$ and edges of $E \backslash M$. Furthermore, an alternating path $\pi$ is augmenting if no edge of $M$ is incident to its extremal nodes. The word "augmenting" means that $(M \backslash \pi) \cup(\pi \backslash M)$ is a matching of size $|M|+1$. It is well known that $M$ is of maximum size on $G$ if $G$ does not admit any augmenting alternating path with respect to $M$.

Let $I$ be an instance of $\mathrm{MOA}_{0,1}$ defined upon $G$. Let $\hat{M}$ be an optimal matching built as follows. Start with the feasible matching $\tilde{M}$ and increase its size with augmenting alternating paths while it is possible.

Let $\hat{M}^{j}$ be the matching produced at step $j$. We suppose that $t$ steps are needed to reach $\hat{M}$. Hence, $\hat{M}^{0}=\tilde{M}$ and $\hat{M}^{t}=\hat{M}$. We mainly prove

$$
w_{i}\left(\hat{M}^{j+1}\right) \geq w_{i}\left(\hat{M}^{j}\right), \forall i \in\{1, \ldots, q\}
$$

for all $j \in\{0, \ldots, t-1\}$. This inequality states that the use of an augmenting alternating path cannot deteriorate the profit of any organization.

Given $v \in V$ and a matching $M$, let $c(v, M)$ be the contribution of $v$ to the profit of its organization in $M$ :

$$
c(v, M)= \begin{cases}p_{s} & \text { if } v \in S \text { and an edge of } M \text { is incident to } v \\ p_{b} & \text { if } v \in B \text { and an edge of } M \text { is incident to } v \\ 0 & \text { otherwise }\end{cases}
$$

Let $V^{\prime}$ be the vertices of $\pi^{\prime}$, the augmenting alternating path such that $\hat{M}^{j+1}=$ $\left(\hat{M}^{j} \backslash \pi^{\prime}\right) \cup\left(\pi^{\prime} \backslash \hat{M}^{j}\right)$. We deduce that

$$
w_{i}\left(\hat{M}^{j+1}\right)-w_{i}\left(\hat{M}^{j}\right)=\sum_{v \in V^{\prime}} c\left(v, \hat{M}^{j+1}\right)-c\left(v, \hat{M}^{j}\right)
$$

for all $i \in\{1, \ldots, q\}$. One can observe that $c\left(v, \hat{M}^{j}\right)=c\left(v, \hat{M}^{j+1}\right)$ if $v \in V^{\prime}$ and $v$ is not an extremal node of $\pi^{\prime}$. Indeed, a buyer $b \in V^{\prime}$ matched with a seller $s \in V^{\prime}$ in $\hat{M}^{j}$ is still matched in $\hat{M}^{j+1}$ but with another seller. Similarly, a seller $s \in V^{\prime}$ matched with a buyer $b \in V^{\prime}$ in $\hat{M}^{j}$ is still matched in $\hat{M}^{j+1}$ but with another buyer. If $v \in S \cap V^{\prime}$ (resp. $v \in B \cap V^{\prime}$ ) and $v$ is an extremal node of $\pi^{\prime}$ then $c\left(v, \hat{M}^{j}\right)=0$ and $c\left(v, \hat{M}^{j+1}\right)=p_{s}$ (resp. $c\left(v, \hat{M}^{j}\right)=0$ and $\left.c\left(v, \hat{M}^{j+1}\right)=p_{b}\right)$. Hence,

$$
c\left(v, \hat{M}^{j+1}\right)-c\left(v, \hat{M}^{j}\right) \geq 0
$$

for all $v \in V$ because $p_{s} \geq p_{b} \geq 0$. Using (2) and (3) we obtain $w_{i}\left(\hat{M}^{j+1}\right)-$ $w_{i}\left(\hat{M}^{j}\right) \geq 0$ for all $i \in\{1, \ldots, q\} . \hat{M}$ is a feasible assignment because $w_{i}\left(\hat{M}^{t}\right) \geq$ $w_{i}\left(\hat{M}^{t-1}\right) \geq \ldots \geq w_{i}\left(\hat{M}^{0}\right)=w\left(\tilde{M}_{i}\right)$ for all $i \in\{1, \ldots, q\}$. In addition, $w(\hat{M})=$ $w\left(M^{*}\right)$ because the algorithm stops when no augmenting alternating path exists. In conclusion, $\hat{M}$ is optimal because $w\left(M^{*}\right) \geq w\left(M_{\text {cont }}^{*}\right)$.

Proposition 1 If EХACTPM is polynomial in bipartite graphs when weights are polynomially bounded, then MOA with 2 organizations is polynomial, for every values of $p_{b}, p_{s}$ such that $1 \geq p_{s} \geq p_{b} \geq 0$ and $p_{s}+p_{b}=1$. 
Proof. Let $p_{b}, p_{s}$ be two rational numbers such that $1 \geq p_{s} \geq p_{b} \geq 0$ and $p_{s}+p_{b}=$ 1 , and let $I=(G, w)$ be an instance of MOA with 2 organizations where $G=$ $(V, E)$. Wlog., assume that $w(e), p_{s} w(e)$ and $p_{b} w(e)$ are integers for every edges $e \in E$ (otherwise, multiplying each weight by the denominator of $p_{b}$ if $p_{b} \neq 0$, we obtain an equivalent instance). Moreover, assume that $\forall e \in E, w(e) \leq P(|V|)$ for some polynomial $P$. Let $R=w\left(M^{*}\right)$ be the value of a maximum matching of $G$. Consider the bipartite graph $G^{\prime}=\left(V^{\prime}, E^{\prime}\right)$ built from $G$ by adding dummy vertices and weights 0 in a such way that any matching of $G$ can be completed into a perfect matching of $G^{\prime}$ with same value. Formally, we add a copy of $K_{|S|,|B|}$ and each new $B$-vertex (resp., $S$-vertex) is completely linked to the $S$-vertices (resp., $B$-vertices) of $G$. Then, each shared edge $e=[u, v] \in E$ is replaced by a path of length $3\left[u, u_{e}\right],\left[u_{e}, v_{e}\right],\left[v_{e}, v\right]$ where $u_{e}, v_{e}$ are new vertices. Remark that either $\left\{\left[u, u_{e}\right],\left[v_{e}, v\right]\right\}$ or $\left\{\left[u_{e}, v_{e}\right]\right\}$ is included in any perfect matching of $G^{\prime}$. Consider the weights $w^{\prime}$ given by: $w^{\prime}(e)=(R+1)^{3} w(e)$ if $e$ is internal to organization $O_{1}$ and $w^{\prime}(e)=(R+1)^{2} w(e)$ if $e$ is internal to organization $O_{2}$. Moreover, if $e=[u, v] \in E$ is a shared edge, then $w^{\prime}\left(\left[u, u_{e}\right]\right)=(R+1) p_{s} w([u, v])$ if $u \in S \cap O_{1}$ and $w^{\prime}\left(\left[u, u_{e}\right]\right)=(R+1) p_{b} w([u, v])$ otherwise (ie., $\left.u \in B \cap O_{1}\right)$. Also, $w^{\prime}\left(\left[v, v_{e}\right]\right)=p_{s} w([u, v])$ if $u \in S \cap O_{2}$ and $w^{\prime}\left(\left[v, v_{e}\right]\right)=p_{b} w([u, v])$ otherwise. Finally, all the remaining edges of $G^{\prime}$ are a weight 0 . It is clear that $G^{\prime}$ is construct within polynomial time and $w^{\prime}$ remains polynomially bounded.

For any matching $M$, we denote by $M_{1}$ (resp., $M_{2}$ ) the restriction of $M$ to organization $O_{1}$ (resp., $O_{2}$ ) and by $M_{\text {shared }}$ the set of shared edges of $M$. Denote by $W_{1}$ (resp., $W_{2}$ ) the contribution of the shared edges of $M$ for organization $O_{1}$ (resp., $\left.O_{2}\right)$. Obviously, we get $w\left(M_{\text {shared }}\right)=W_{1}+W_{2}$ since $p_{s}+p_{b}=1$.

We claim that the weight of $M$ is $w(M)=w\left(M_{1}\right)+w\left(M_{\text {shared }}\right)+w\left(M_{2}\right)$ if and only if there exists a matching of $I^{\prime}$ of weight $W=(R+1)^{3} w\left(M_{1}\right)+$ $(R+1)^{2} w\left(M_{2}\right)+(R+1) W_{1}+W_{2}$. Moreover, $M$ is a feasible solution of MOA iff $w\left(M_{i}\right)+W_{i} \geq w_{i}(\tilde{M})$ for $i=1,2$.

One direction is trivial. So, let $M^{\prime}$ be a matching of $I^{\prime}$ with value $w^{\prime}\left(M^{\prime}\right)=$ $W=(R+1)^{3} A+(R+1)^{2} B+(R+1) C+D$. By the choice of $R$, we must get $w_{1}\left(M^{\prime}\right)=A, w_{2}\left(M^{\prime}\right)=B$ and $w\left(M_{\text {shared }}^{\prime}\right)=C+D$, where $C$ (resp., $\left.D\right)$ is constituted by the contribution of the shared edges of $M^{\prime}$ for organization $O_{1}$ (resp., $O_{2}$ ). The profit of $M^{\prime}$ for organization $O_{1}$ is $A+C$ and $B+D$ for organization $\mathrm{O}_{2}$.

In conclusion by applying at most $R^{4}$ times the polynomial algorithm for EXACTPM, we find an optimal solution of MOA. By an exhaustive search, we try all values of $A, B, C, D$ at most equal to $R$ such that $A+C \geq w_{1}(\tilde{M})$ and $B+D \geq w_{2}(\tilde{M})$.

Proposition 2 MOA with a constant number of organizations can be solved within pseudopolynomial time when the underlying graph has a maximum 2.

Proof. Here, we deal with 2 organizations, but the result can be extended to any constant number of organizations. The proof is based on Proposition 1, and use the pseudopolynomiality result of [2] for ЕхАСтPM in planar graphs. However, the construction of $G^{\prime}$ is slightly different because when one add a 

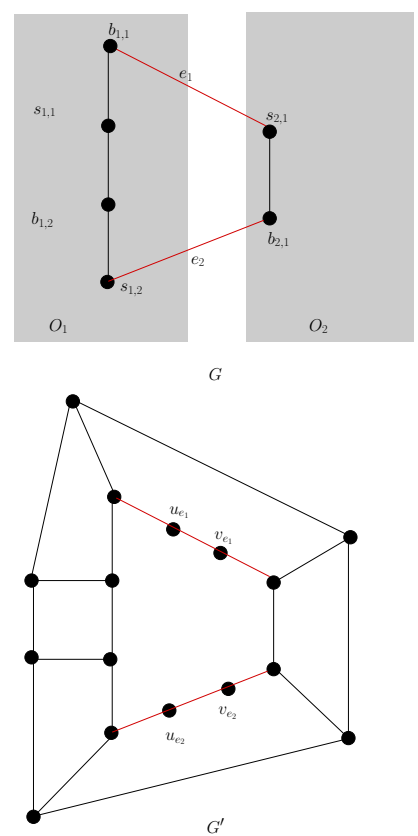
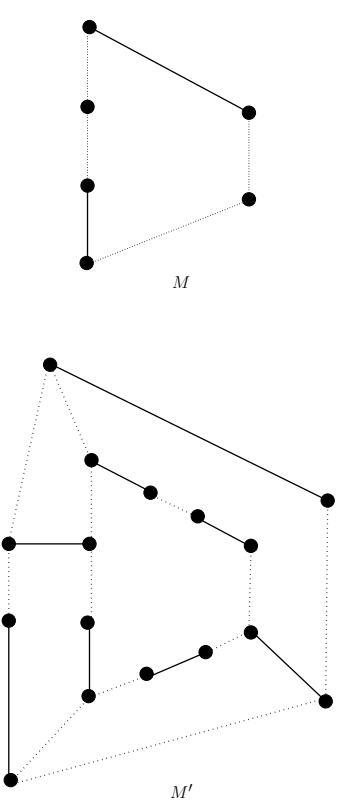

Fig. 4. Construction of $G^{\prime}$ and perfect matching $M^{\prime}$ from $G$ and matching $M$.

copy of $K_{|S|,|B|}$ the resulting graph may be not planar. So, let $I=(G, w)$ be an instance of MOA with 2 organizations where $G=(V, E)$ is a bipartite graph of maximum degree 2. Wlog., assume that $G$ is 2-regular, that is a collection of disjoint even cycles (by adding dummy vertices and edges of weight 0 ). Then, for each cycle $C$ of $G$, we add a copy $C^{\prime}$ of $C^{\prime}$ and we link each vertex of $C$ to its copy in $C^{\prime}$. Finally, as it is done in Proposition 1, each shared edge $e=[u, v]$ of a cycle $C$ in $G$ is replaced by a path of length $3\left[u, u_{e}\right],\left[u_{e}, v_{e}\right],\left[v_{e}, v\right]$ where $u_{e}, v_{e}$ are new vertices. The weights are defined similarly to the one given in Proposition 1. Figure 4 gives an illustration of this construction.

Obviously, $G^{\prime}$ is planar. Moreover, any matching $M$ can be converted into a perfect matching $M^{\prime}$ of $G^{\prime}$. Thus, by applying the argument given in Proposition 1 , the result follows.

Property 2. $w_{i}\left(M^{*}\right) \geq p_{b} w\left(\tilde{M}_{i}\right)$, and this bound is asymptotically tight.

Proof. Let $C_{i}$ be the set of edges of $M^{*}$ whose at least one endpoint belongs to organization $O_{i}$. We have $w\left(C_{i}\right) \geq w\left(\tilde{M}_{i}\right)$, otherwise we could obtain a matching of weight larger than $w\left(M^{*}\right)$ by replacing the edges of $C_{i}$ by the ones of $\tilde{M}_{i}$. The profit of $O_{i}$ is $w_{i}\left(M^{*}\right) \geq p_{b} w\left(C_{i}\right)$, and thus $w_{i}\left(M^{*}\right) \geq p_{b} w\left(\tilde{M}_{i}\right)$.

Let $\varepsilon$ be a small positive number. Let us now show that the above bound is tight, by considering the following instance : two organizations $O_{1}$ and $O_{2}$, such that there are in $O_{1}$ two nodes $b_{1,1}$ and $s_{1,1}$ linked by an edge of weight $1-\varepsilon$, and there is in $O_{2}$ one node $s_{2,1}$ linked to $b_{1,1}$ by an edge of weight 1 . We have: 
$w\left(\tilde{M}_{1}\right)=1-\varepsilon, M^{*}=\left\{\left[b_{1,1}, s_{2,1}\right]\right\}$, and $\frac{w_{1}\left(M^{*}\right)}{w\left(\tilde{M}_{i}\right)}=\frac{p_{b}}{1-\varepsilon}$, which tends towards $p_{b}$ when $\varepsilon$ tends towards 0 .

Proposition 3. The price of stability is $p_{b}$.

Proof. It follows from Theorem 4 that $w\left(M_{\text {cont }}^{*}\right) / w\left(M^{*}\right) \geq p_{b}$ since ApProx returns a matching $M$ such that $w\left(M_{\text {cont }}^{*}\right) \geq w(M) \geq p_{b} w\left(M^{*}\right)$.

Let us now show that this bound is tight. There are two organizations: organization $O_{1}$, which owns two vertices $b_{1}$ and $s_{1}$, linked by an edge of weight $W_{1}$, and organization $O_{2}$, which owns one vertex $s_{2}$, linked to $b_{1}$ by a link of weight $W_{2}$. Suppose that $W_{1}=\varepsilon$ such that $0<\varepsilon<1$ and $W_{2}=1$ when $p_{b}=0$. The ratio $\frac{w\left(M_{\text {cont }}^{*}\right)}{w\left(M^{*}\right)}=\varepsilon$, tends towards $0=p_{b}$ when $\varepsilon$ tends towards 0 . Suppose that $W_{1}=1$ and $W_{2}=1 / p_{b}-\varepsilon$ such that $0<\varepsilon<1 / p_{b}-1$ when $p_{b}>0$. The ratio $\frac{w\left(M_{\text {cont }}^{*}\right)}{w\left(M^{*}\right)}=\frac{p_{b}}{1-\varepsilon p_{b}}$, tends towards $p_{b}$ when $\varepsilon$ tends towards 0 . 